\title{
أشكال الاتجاهات في تعليم اللغة العربية لدى قسم تعليم اللغة العربية بجامعة باتوسنكر الإسلامية الحكومية
}

\author{
Muhammad Husni Shidqi \\ e-mail: shidqie11@gmail.com \\ Institut Agama Islam Negeri Batusangkar
}

ملخص: يتناول هذا البحث بدراسة تدور حول أشكال الاتجاهات في تعليم اللغة العربية لدى قسم تعليم اللغة العربية في جامعاة باتوسنكر الإسلامية الحكومياة. و وصف الباحث هذه المشكلات و حللها تحليلا متكاملا لإنجاز تعليم اللغة العربية على المستوى الجامعي في قسم تعليم اللغة العربية هذه الجامعة. واستخدم هذا البحث المدخل الكيفي والمنهج الوصفي بدراسة الحالات المات المتعددة وللحصول على البيانات استخدم الباحث المقابلة والملاحظة و دراسة الوثائق لمتعلقة بهذا البحث. أما نتائج البحث فهي أنّ أشكال الاتجاهات المات لتعات التعليم

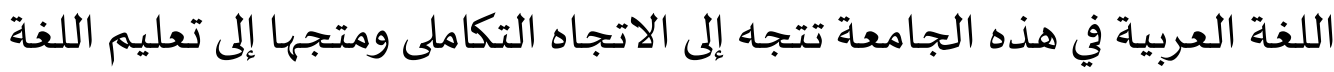

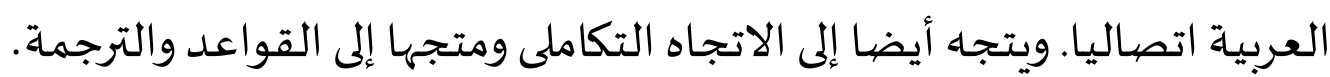
الكلمات الأسـاسية: اتجاه التعليم، الاتجاه التكاملي، اللغة العربية، الجامعة الإسلامية.

\begin{abstract}
This research is based on trends of orientation in Arabic language learning in the Department of Arabic Language Education of IAIN Batusangkar. The researcher wanted to describe these problems and did integrated analysis to complete the teaching of Arabic language at university level in the Department of Arabic Language Education in this college The researcher implemented descriptive qualitative design, the cases (multi-sites) of which were studied through observation, interview, and related documents to obtain the data.This research found that the learning system of Arabic language at LAIN Batusangkar was oriented not only to integrative orientation as based learning, but also to integrative orientation but focus on learning grammar translation method.
\end{abstract}

Key words: Learning orientation, integrative orientation, Arabic language, State Islamic University. 
الأشكال من الفتحة والكسرة والضمة

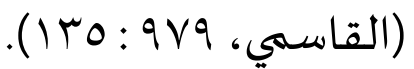

أما الطريقة الشائعة المطبقة للوصول إلى هذا الهدف فهي طريقة

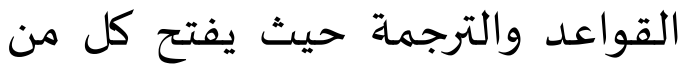
المدرس والمتعلم الكتاب المقرر فيقراً المدرس الكتاب ويترجم ما فيه كلمة فكلمة إلى اللغة الإقليمية أو اللغة المحلية، بينما المتعلم يكتب شرح الماه

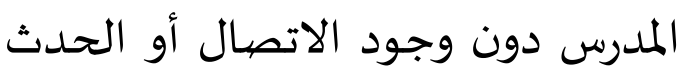

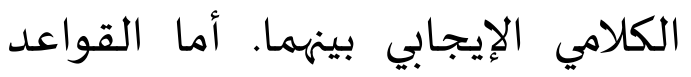
فتدرس عن طريق شرح موقع الكلمات

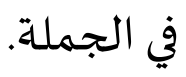

إن اللغة العربية هي لغة العروبة والإسلام وهي أعظم مقومات الأمة الإسلامية منذ أن بعث الله رسوله العربي إلى أن يرث الله الأرض ومن عليها.

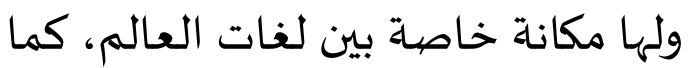

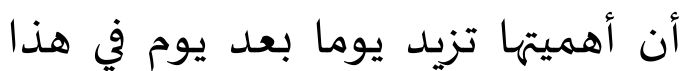

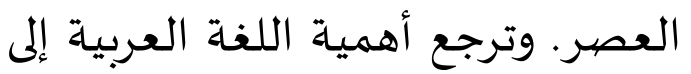

$$
\text { الأسباب الآتية : }
$$

1. إنها لغة المصيدرين الأسـاسيين لدين الاسين الإسلام الحنيف (القرآن الكريم والحديث النبوي) وهي بذلك، اللغة الاسلةم التهريه التي يحتاج إلهها كل مسلم ملتزم

$$
\text { بشرائع دينه }
$$

وصلت اللغة العربية إلى إندونيسيا منذ دخول الإسلام إلى هذه الدولة في القرن الأول الهجري الموافق بالقرن السابع الميلادي، استنادا إلى السجلات الى بهري

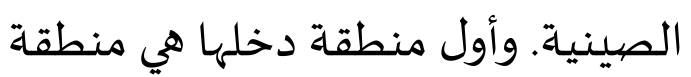

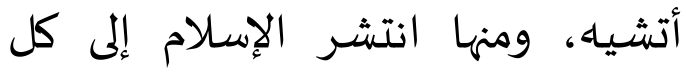
الولايات الموجودة في جنوب شرق آسيا

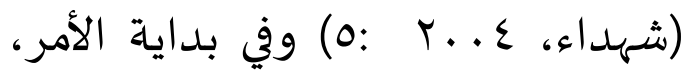
كان الهدف من تعلم الإندونيسيين المسلمين هذه اللغة وتعليمها ليس إلا لإنائ

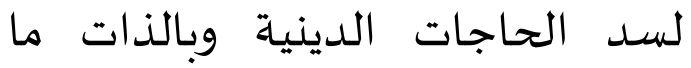
يتعلق بأداء العبادات الدينية كالصلاة. فالمواد اللغوية المدروسة محصيورة في الأدعية والأذكار والسور القصيرة من المن القرآن. أما الطريقة المتبعة في هذا لادها التعليم فهي الطريقة الأبجدية (alphabetic method)

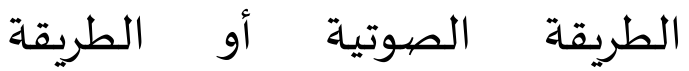
البغدادية. ركزت هذه الطرق -مع الطيه اختلاف تسميتها- في قراءة أحرف القرآن

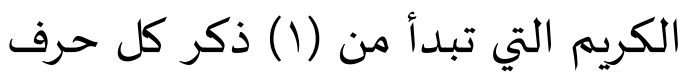
باسمه مرتبا، (Y) تعليم المفردات المتكونة من حرفين ثم ثلاثة عن طريق مرنا، التدرج، ثم (r) تعليم الحركات أو مرين لهن 
r. أنّ نتيجة الاعتماد لقسم تعليم

اللغة العربية هي درجة الامتياز (A)

r. مشاركة الطلاب وإنجازهم في بعض درديه

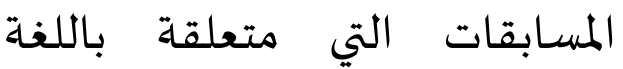

العربية إماّ في المستوى المنطقي

$$
\text { والوطني. }
$$

ع. عقد البرامج اللغوية كالعرض الفني والمسرحي لتنمية اللغة العربية.

0. قدرة الطلبة على إجادة المهارات

اللغوية الأربع ويطبقون هذه المهارات

في العمليات التعليمية باستخدام

$$
\text { الطريقة التواصلية. }
$$

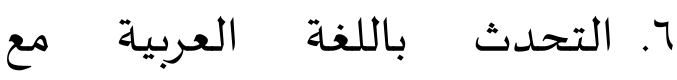
أصدقائهم داخلا أو خارج الفصل

$$
\text { الدراسي. }
$$

V. قدرة الطلبة على ترجمة النصوص

$$
\text { المقررة في الدراسـة. }
$$

تعريف اتجاه التعليم

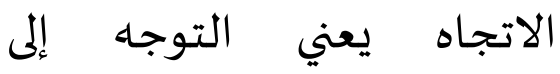

المستقبل وعن شيء جديد. وهذا أمر المر

هام متعلق بمختلف الظروف القائمة والأحداث والفرصة التي تفتح في حياة

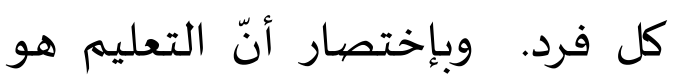

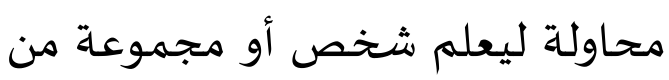

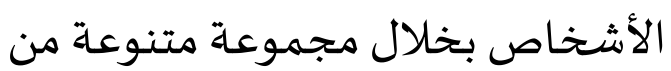
الجهود والإستراتيجيات والطرائق متئن r. إنها لغة العبادة أي أن بعض

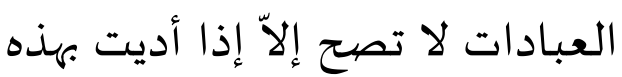
اللغة كالصلاة. r. المكانة الاقتصادياة للعرب. إن العرب العبده

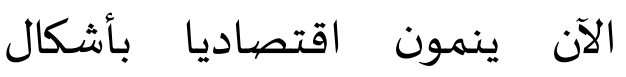

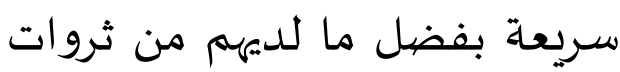
نفطية ومعدنياة، مما يجعل لهم وزنا اقتصاديا كبيرا، ووزنا سياسيا

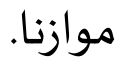
ع. عدد متكلمي اللغة العربية. إن سبع دول العالم تتكلم بها كلغة أولى كما أن كثيرا من شعوب الدول الاسلامية تستخدمها كلغة ثانياة (الخولي، .$(v-7: 1919$ إنّ جامعاة باتوسنكر الإسلامية الحكومية تقع في منطقة تانه داتر بسومطرة الغربية، وقد تمت ولم تزل ترقية تعليم اللغة العربية في هذه الهونه الجامعة منذ بداية إنشائها ترقية ملموسـة. و من خصائص تعليم اللغة العربية في مانئ الجامعة ما يلي :

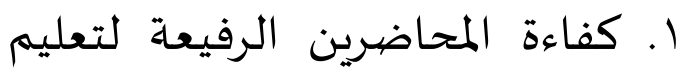
اللغة العربية، وذلك لأنّ معظم محاضرين في تعليم اللغة العربية قد حصلوا على درجة الدكتور اه. 
والصرف والبلاغة والمطالعة والمفردة

والنصوص الأدب وغير ذلك. إنّ تعليم والمهرده اللغة العربية التامّة يقدر على إيصال الطلبة على استيلاء المهارات اللغوية بجانب إستيلاء على العلوم التي ذكر اسلراء المهات التوبه قبله.

و ظهرت أربع الاتجاهات في تعليم اللغة

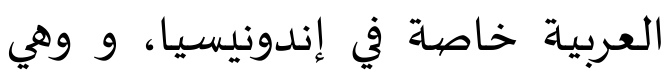

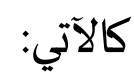

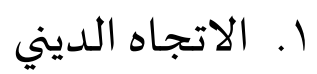

مهدف تعليم اللغة العربية إلى تهمياه فهم و تفهيم شريعة الإسلام، وهذا الاتجاه يكون من تعلم المهارة

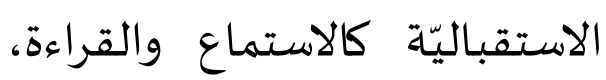
وكذلك يكون من تعلم المهارة الإنتاجية كالكلام و الكتابة. r. الاتجاه التعليمي أو الأكاديمكي الإنتاهي هو الاتجاه في تعلم اللغة العربية الذي يهدف إلى فهم العلوم والمهارات اللغوية، ومكانة اللغة العرية على سبيل هذا الاتجاه وهي إحدى العلوم اللازمة و الدراسة الدية التي لا بد من إستيلاءها. ا. الاتجاه المهني هو تعلم اللغة العربية من أجل

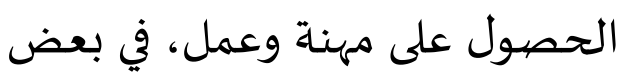

والمدخل نحو تحقيق الأهداف التي

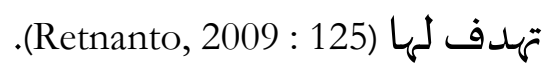

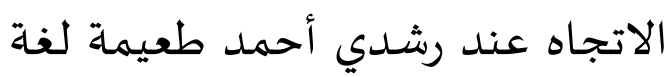
يعني القصد و الإقبال نحو شيء معين. يقال اتجه فلان إلى البيت. أي جعل الئل البيت وجهته أي قصيده وأقبل عليه.

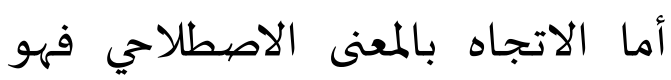

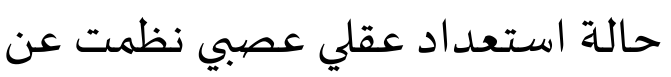
طريق التجارب الشخصية وتعمل على توجيه استجابة الفرد لكل الأشياء والمواقف التي تتعلق بهذا الاستعداد ( طعيمة و المناع، ( . . Y: 9).

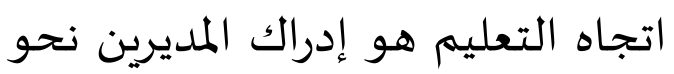
الالتزام التنظيمي بأهمية التعليم في التهاه المنظمة، والانفاق في الرؤية، وانفتاح

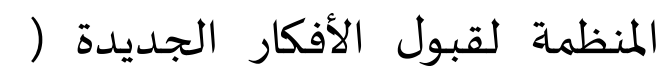
453: Sutanto, 2009 و و اتجاه التعليم هو فلسفة تعتقدها الشركات التي تركز على التعليم في المنظمة. وسوف يطور

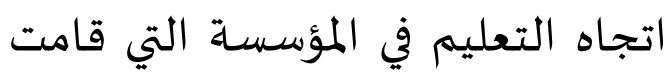
بالدراسة. ستحدث في منظمة الموجهة نحو التعليم وتجري عملية التطوير المهارات بشكل مستمر من أجل عقد التعليه وتجري عمله التطوير أحسن المستقبل. احتوى علم اللغة العربية إلى المستعبل عد من العلوم ومن بينها: علم النحو احتوى لعربه العيه 
الإتصال في المجتمع الذي استخدام

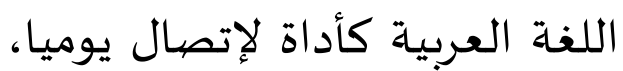
بمعنى أنّ لابد لكل فرد في الاجتماع على التكلم والتواصل باللغة العربية بنشاط مستمر. وبالممكن أن تفضي الحالة على سبيل المثال بين شخصين متعلمين في المحادثة مباشرة، واتصلت الفكرة بالكلمات التي ظهر في تعبير وسيمائهم والبيئة فيها حتى تكون عملية التعليم فعالة. ولكن تعليم اللغة العربية لايكون

فعالة إذا ما سـاعد المجتمع بالبيئة التي تستخدم اللغة العربية كأداة للتواصل اليومي. وطلب المدرسين الذين قاموا بتطبيق منهج لتعليم اللغة العربية أن يقدموا المواد

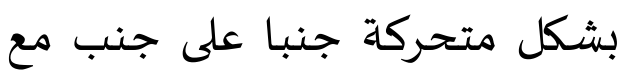
حركة التطوير اللغة العربية المستخدمة مع الناطق الحقيقي زمنيا. r. منهج التعليم المتجه إلى القواعد منهج تعليم اللغة العربية الذي الذي الفي مبني على الافتراض القائل بأن اللغة هي معايير أو قواعد اللغة مأخوذة من نصوص فصدح. و توجد في اللغة العربية نصوص القرآن و الحديث و
الدول العربية و من أجل التجارة والالتحاق بالدراسة الجامعية في دول الشرق الأوسط. r. الاتجاه الفلسفي و الاقتصادي يعني تعلم اللغة العربية ليكون

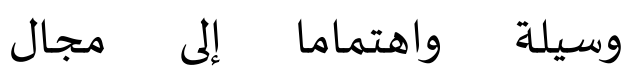
الاستشراق والاستعمار والرأسمالي. و الدليل على هذا الاتجاه يعني بافتتاح المؤسسات الدورية في تعليم Mujib, اللغة العربية في دول أوربا .2010: 84-85)

قالت أم هجرية في بحثها إنّ المنهج

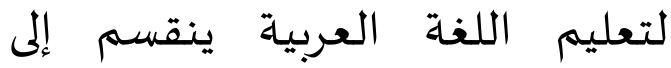

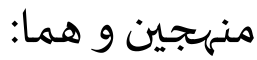
1. منهج التعليم المتجه إلى إستيلاء اللغة إتصاليا (للمحادثة) منهج تعليم اللغة العربية يستند

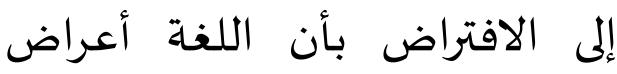

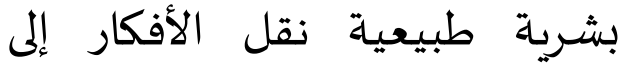
الآخرين أو قبول أفكار الآخرين. وبعبارة أخرى، أنّ الإنسان ككائن

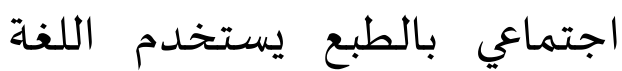

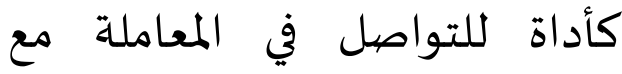
الآخرين. إنّ منهج تعليم اللغة العربية أكثر فعالية للطلبة في إنيات إستيلاء اللغة العربية أداة 
الأطفال يتعلمون القراءة بغرض

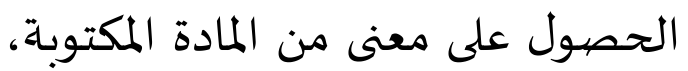
وهم يتفاعلون معها لتكوين نشاط له اله ادهي

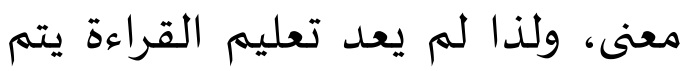

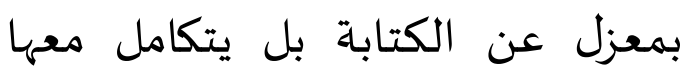

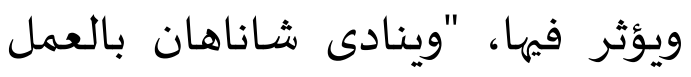
على تكامل مهارات اللغة من استماع وتهاع وتحدث وقراءة وكتابة في البرنامج من الفهارئ

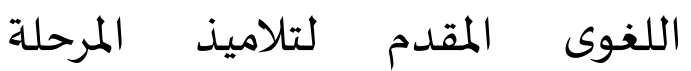
الابتدائية، ويرى الناقة أن تنمية أية

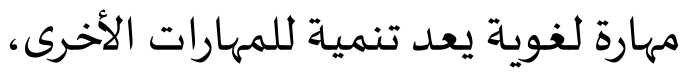
وأن يتم اكتساب القواعد النحوية من فئن خلال استخدام مهارات اللغة المختلفة بحيث يتكامل معها بشكل وظيفى، فهو

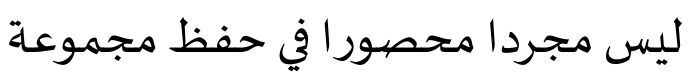
من القواعد دون القدرة على تطبيقها مجردا مجها واستخداهها. وفي دراسة لكل من نلسون عن اتجاهات تعليم القراءة والكتابة

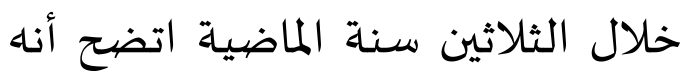
من بين هذه الاتجاهات التركيز على آلى

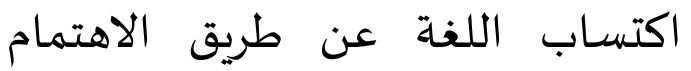
بمهارات الاستماع والتحدث والقراءة التراء والكتابة حيث تكمل كل من تلك

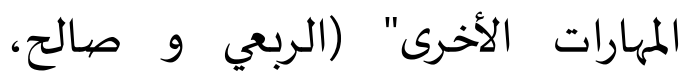
(r). T. T . T
الكتب التي تتركز فيما يتعلق بالقواعد. ووفقا لهذه الافتراضات بمن يريد أن يدرس القرآن،

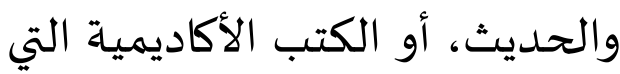
لها تركيز قوي في القواعد، ووجب عليه أن يتم السيطرة على قواعد فيد اللغة العبية (Hijiriyah, 2010).

\section{الاتجاهات الحديثة في تعليم اللغة العربية} اتجاه تكامل مهارات اللغة ينبثق هذا الاتجاه من المداخل الحديثة في تعليم اللغة الأم و بخاصية في من الماهي المرحلة الابتدائية، وهو يعتمد على نتائج

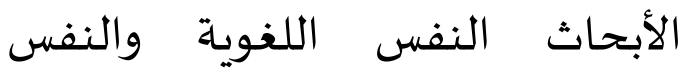
الإجتماعية وهو يؤكد الصلة الوثيقة بين الفنون اللغوية المختلفة حيث إن تعليم إحدى المهارات يؤثر بالضيرورة في

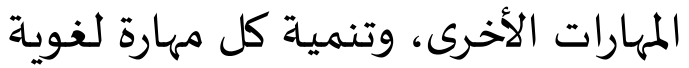

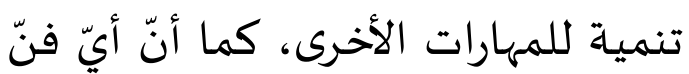
منها لا يمكن فهم طبيعته بدون طبيعة

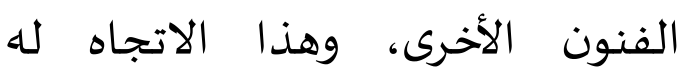
تطبيقات عديدة في تعليم اللغة وتطوير الاستراتيجيات و وفنيات و وإجراءات وطرائق تدريسهامما جعل الدراسات

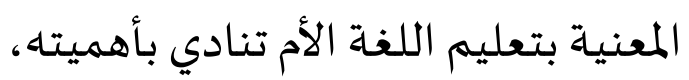

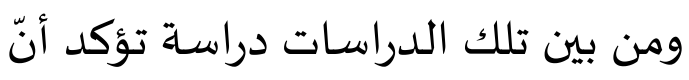


المواقف التي تتطلب استخدام الفنون اللغوية المختلفة، وكلما كانت تلك المواقف حية ويحرص المعلم على توفيرها للأطفال من خلال الأنشطة اللغوية المختلفة، فإن النمو اللغوى للطفل يتقدم بشكل جيد وقد أكدت دراسـة على ضرورة تحقيق التكامل بين الأنشطة اللغوية المختلفة مثل أنشطة التحدث والاستماع والقراءة والكتابة والأنشطة الأدبية ويمكن أن تتضمن تلك الأنشطة مختارات من أدب الأطفال لاتخاذها محورا للدروس، ومن ثم فإن التحدى الأكبر لمعلمى اللغة يتمثل في اختيار الأنشطة اللغوية التي توفر الخبرات اللغوية لألمتنوعال، وتسـاعدهم على ممارستها "فالكلمات التي يستمع إليها التلميذ ويفههمها هي التي يمكن أن يستخدمها في حديثاه وسستعين بها في فهم ما يقرأ

$$
\text { ويستخدمها في كتابته". }
$$

r. تكامل المهارات (Skill Integration)

إن التكامل في تعليم اللغة يعنى - كما يؤكد النظر إلى اللغة على أهنا وحدة متكاملة ليس هنالك قراءة
ثم أوضحت دراسـة معاطى محمد و إبراهيم نصر أن المعلم يستطيع تحقيق التكامل بين فنون اللغة بعدة طرق منها:

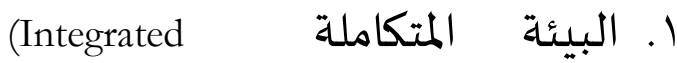
Environment) ويقصد بتلك البيئة بيئة المدرسـة والفصل حيث يجب وضع التلاميذ في بئة مدرسية ثرية متكاملة مملوءة بالأنشطة اللغوية التي تتناسب مع ميولهم واهتماماتهم مع إتاحة الفرصية للتفاعل الصفى والتواصل الهادف مع استخدام المصادر التعليمية المتنوعـة لإثراء عمليات المناقشة والحوارات، ولإتاحة الفرصة كذلك لتبادل المعلومات بمختلف الوسائل المقروءة والمسموعة والمكتوبة، وتؤكد الدراسـات على أهميـة بيئة الفصل في تحقيق التكامل بين فنون اللغة في الصفوف الدراسية المختلفة ومن تلك الدراسـات دراسـة. r. Integration الأنشطة Activities)

تؤكد الدراسـات المعنية بتعليم اللغة أن الطفل يواجه داخل

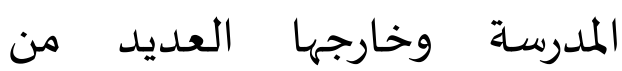


الأعمق لها. ويسير هذا البحث على المنهج الوصفي بدراسـة الحالة المتعددة لهاتيات

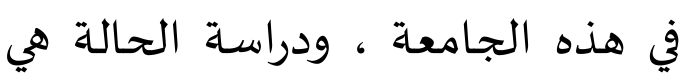
دراسة مظهر ما من مظاهر السلوك

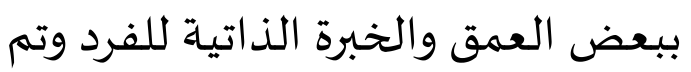
ذلك عن طريق جمع بيانات كيفية وصفية تفصيلية عن ذلك الشخص باستخدام المقابلة والملاحظة أو كلاهما

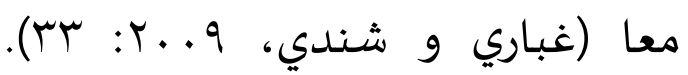
وعندما درس الباحث اثنين أو أكثر من

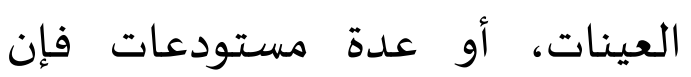
المعلومات التي قام بدراستها عادة ما عاديتودات فان يسمياه دراسة الحالة المتعددة. أما مصيادر البيانات على حصول دراسلهالمتودة. البيانات فتنقسم إلى نوعين: المصيدر الأساسي والمصيدر الثانوي (عبيدات و ولئات ناصر، 1999: الكيفية هي البيانات التي تحتوي على جودة موضوع البحث. فتمثل بياناتها في صورة الألفاظ والسلوكيات ولا بالأرقام والحسابات.

والبيانات المحتاجة لهذا البحث

تشتمل على الملاحظة في اتجاه تعليم اللغة العربية باعتبارها بيانات أساسية ، وكذلك المقابلة مع رئيس قسم تعليم اللغة العربية والمحاضرون فيها،
وحدها وليس هناك كتابة وحدها..فالشخص حينما يستخدم اللغة فإناه يستخدمها بصورة كلية بمعنى أنه حين يرسلها كلاما وكتابة فإناء يستخدم كل ما تعلماه من لغةلة وكذلك حين يستقبلها استماعا وقراءة، فإنه يستخدم كل ما تعلماه من مفردات وتراكيب ودلالات

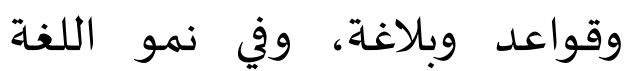
نلاحظ أن الاستماع هو الباب الرئيسى لإنتاج اللغة...وعلاوة على الى العاب ذلك فالحديث أو الكلام إذا كان ثريا، فكرا وأسلوبا من دلائل استعداد الطفل لتعلم القراءة والكتابة. وقد أثبتت الدراسات أن الكتابة جماع كل الفنون اللغوية وأن الشخص الذي لا يكتب جيدا لا يقرأ أصلا قراءة جيدة، علاوة على ضحالة ثروته اللغوية حين يتحدث وعدم قدرتاه على فهم ما يستمع إليه

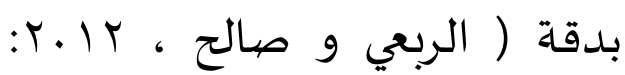
. (rRt طريقة البحث المدخل المتبع في هذا البحث هو

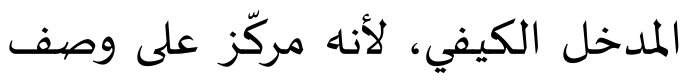

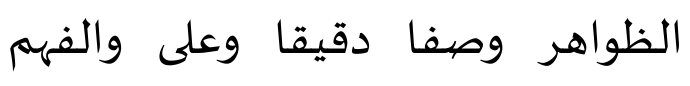


اللغة العربية. والملاحظة التي يقوم

بها الباحث هي الملاحظة بالمشاركة.

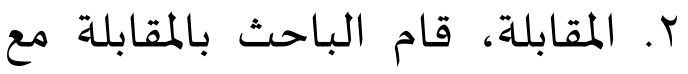

رئيس قسم تعليم اللغة العربية

والمحاضرين فيها عمّا يتعلق بأشكال

الاتجاهات في تعليم اللغة العربية.

r. الوثائق، قام الباحث بت بتحليل

مضمون الوثائق الموجودة في قسم

تعليم اللغة العربية، و أشكال

الاتجاهات وما يرتبط بالموضوع.

قام الباحث قبل تحليل البيانات

بتثليث (triangulasi) البيانات، وقسّم دينزين

هذه التقنية -كما قال وفئال (Denzin)

موليونج- إلى أربعة أقسام : التثليث

بالمصيادر والتثليث بالطريقة والتثليث

بالباحث والتثليث بالنظرية. والتثليث

الذي استخدماه الباحث في هذا البحث

هو التثليث بالمصيادر والتثليث

بالطرائق.

التثليث بالمصيادر في سياق هذا

البحث يقوم الباحث بمقارنة البيانات

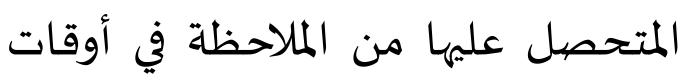

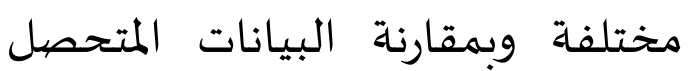

عليها من مقابلة مع رئيس قسم تعليم

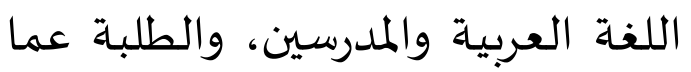

تتعلق بتعليم والمحاولات لتعليم اللغة
والبيانات الثانوية هي البيانات المتحصلة من الوثائق والكتب البيئة اللغوية المؤثرة في تعليم اللغة العربية في قسم تعليم اللغة العربية.

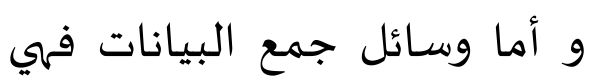
كالآتي: 1. الملاحظة، قام الباحث بالماحظة في نظر أشكال الاتجاهات في تعليم اللغة العربية بالجامعةة. والبيانات المأخوذة تدور حول تاريخ الجامعة.

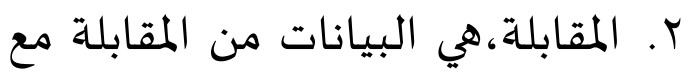
رئيس قسم تعليم اللغة العربية والمحاضرون فيها عمّا تتعلق بأشكال الاتجاهات في تعليم اللغة العربية.

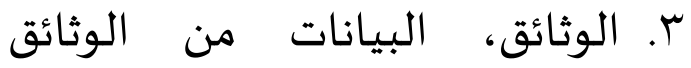
الموجودة في قسم تعليم اللغة العربية، وثيقة تاريخ تأسيس قسم تعليم اللغة وأشكال الاتجاهات والكتابة العربية وأنواع البيئة اللغوية أما أساليب جمع البيانات في هذا

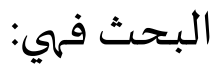
1. بالملاحظة المتعمقة، قام الباحث بالملاحظة . المرات لكل الجامعة في نظر عن أشكال الاتجاهات في تعليم 
فكانت الخطوات التي نفذها الباحث

اعتمادا على الأنماط المذكورة كمايلي:

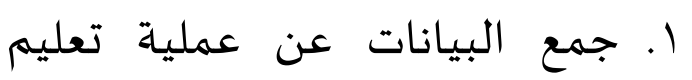
المهارات اللغوية و الأنشطة اللغوية وأشكال الاتجاهات في تعليم اللغة العربية وتفتيشها

r. تفصيل البيانات، يقوم الباحث

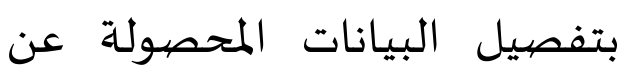
عملية تعليم المهارات اللغوية

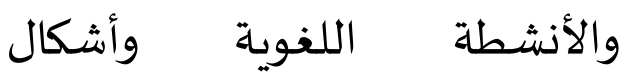
الاتجاهات في تعليم اللغة العربية، ويأخذ البيانات المتعلقة بالموضوع ويهمل ما لا يتعلق بالبحث.

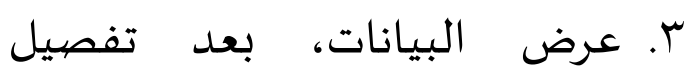
البيانات، يقوم الباحث بالتعيين،

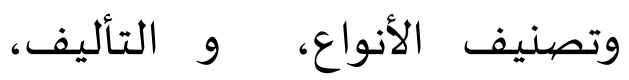

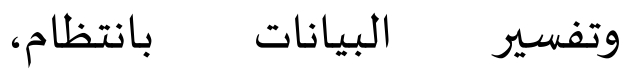

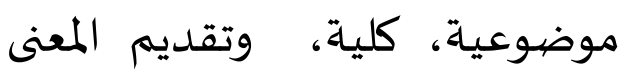
عمّا يتعلق بعملية تعليم المهارات اللغوية والأنشطة اللغوية وأشكال الاتجاهات في تعليم اللغة العربية. ع. الخلاصة عمّا تتعلق بعملية تعليم المهارات اللغوية والأنشطة اللغوية وأشكال الاتجاهات في تعليم اللغة العربية : Miles \& Huberman, 1992
العربية بجامعة باتوسنكر الإسلامية الحكومية لنظر عن أشكال الاتجاهات

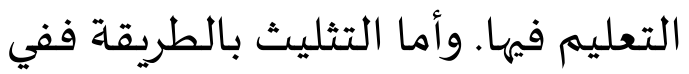
هذا البحث قد قام الباحث بمقارنة البيانات المتحصل عليها من المقابلة بالبيانات المتحصل عليها من الملاحظة

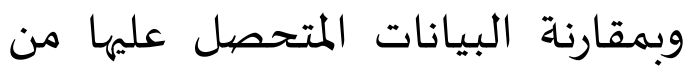
المقابلة باليبانات المتحصل عليهاتهات المتحصل علها من من الوثائق المكتوبة بعد أن تناول الباحث البيانات في الجامعة. وتحليل البيانات

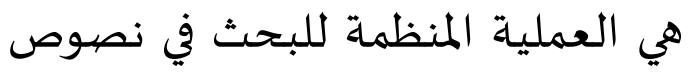
المقابلات والملاحظات الميدانية والمواد الأخرى التي جمعت من خلالها البيانات وتنظيمها عما تتعلق بعملية تعليم المهارات اللغوية والأنشطة اللغوية وأشكال الاتجاهات في تعليم اللغة العربية في الجامعة ولزيادة فهم الباحث لها ولتمكين من تقديم ما اكتشفه للآخرين (غباري و شندي، 9 ـ . ب: عـ). وذهب الباحث عند وصيول الباحث لمرحلة التحليل القائم بتصنيف بنديف البيانات النوعية عند استخدام دراسة التهائ الحالة بطريقة تسهل عليه الوصول

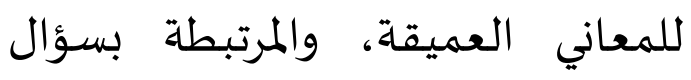
البحث وتنظم آلية عرضها. 
وإنتاج علماء اللغة العربية

التعليمية القادرة على تصميم تعلم اللغة العبية وإدارها وتطويرها وأيضا إنتاج علماء اللغة العربية التعليمية المختصة بإجراء البحوث في مجال اللغة العربية وتعاليماه وإنتاج المتخرجين لتعليم العربي الإسلامي وشخصية إندونيسيا. ع. منهج التعليم اللغة العربية أنّ المنهج لتعليم اللغة العربية في هذه الجامعة مؤسسا على المنهج لتكوين المرشحين في تعليم (KKNI) اللغة العربية، يرى أن ترتيب المواد في إدارة قسم تعليم اللغة العربية في هذه الجامعة يتكون من ثلاثة

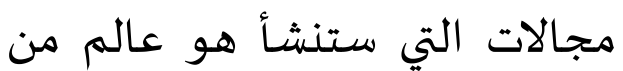
القيم أو المواقف (العاطفية)، عالم المعرفية (المعرفة).

قالت أم هجرية في بحثها أنّ المدخل لتعليم اللغة العربية يتجاه

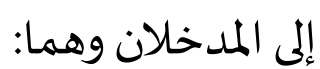

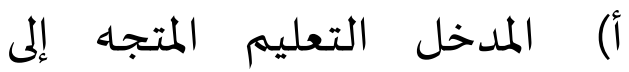
إستيلاء اللغة إلتصاليا (للمحادثة)

هو المدخل تعليم اللغة العربية يستند إلى الافتراض لمدر لهيم المه
نتيجة البحث ومنقاشتها

إن اتجاهات التعليم في قسم تعليم اللغة العربية في جامعاة باتوسنكر الإسلامية الحكومية يمكن

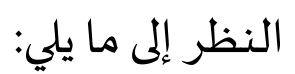

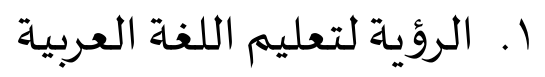
إنّ الرؤية لتعليم اللغة العربية في الجامعة هي أن يصبح قسم

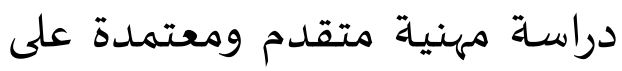
التكنولوجيا والبحوث الحديثة. r. الرسالة لتعليم اللغة العربية إنّ الرسالة لتعليم اللغة العربية في هذه الجامعة هي إدارة التعليم

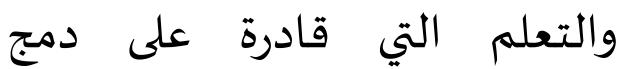
المعلومات العربية والإسلامية من العلم و و التكنولوجيا والقدرة النفسية وتنظيم التعليم والتعلم على دراسة المهارات التي تجهز لنجاح في اللغة العربية وتعليماء. r. أهداف تعليم اللغة العربية

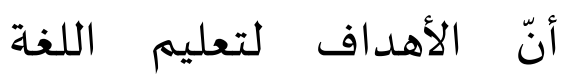
العربية في هذه الجامعة هي إنتاج المتخرجين الذين إستولوا اللغة العربية والعلوم لتدريس اللغة إستونوين العربية وتكامل العلوم الإسلامية التي تدعمها تكنولوجيا المعلومات 
ولكن تعليم اللغة العربية

لايكون فعالة إذا ما ساعد

المجتمع بالبيئة التي تستخدم

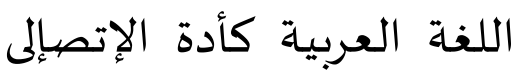

اليومياة. وطلب إلى المدرسين

الذين قاموا بتطبيق المدخل

لتعليم اللغة العربية أن الن الن الماين

يقدموا المواد بشكل متحركة

جنبا على جنب مع حركة

التطوير اللغنة

المستخدمة مع الناطق

$$
\text { الحقيقي زمنيا. }
$$

ب) المدخل التعليم المتجها إلى زمنيا. القواعد

هو المدخل تعليم اللغة

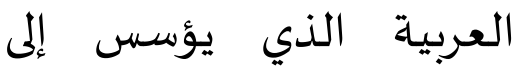

الافتراض بأن اللغة هي معايير

أو قواعد اللغة مأخوذة من لن

نصوص الفصيح. وفي اللغة ماحودة من

العربية كمثل من نصيوص

القرآن والحديث والكتب التي

تتركز فيما يتعلق بالقواعد.

ووفقا لهذه الافتراضات بمن بـن بـن

يريد أن يدرس القرآن،

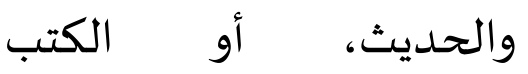

الأكاديمية التي لها تركيز قوي الودية
بأن اللغة أعراض بشرية

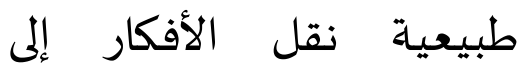
الآخرين أو قبيدئ أفكار الآخرين. وبعبارة أخرى، أنّ الآن الإنسان كالبشر الاجتماعية وبعاره احري، ان

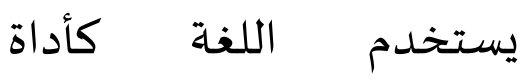

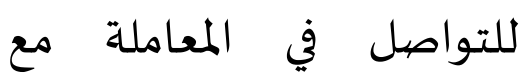

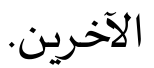

أنّ المدخل تعليم اللغة العربية أكثر فعالية للطلبة في إستيلاء اللغة العربية كأداة

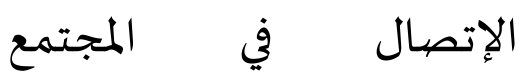
الاجتماعية الذي استخدام اللغة العربية كأداة لإتصال الإيكال يوميا، بمعنى أنّ لابد لكل فرد في الاجتماع على التكلم و ولائى

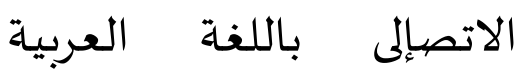
بنشاط مستمرا. وبالممكن أن بالن النمريك تفضي الحالة على سبيل المثال بين شخصين متعلمين في المحادثة مباشرة، واتصلت الفكرة بالكلمات التي ظهر في تعبير وسيمائهم و البيئة فيها حتى تكون عملية التعليم

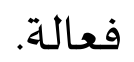


وقال ليتيلوود أنّ هذا المدخل

الذي يدمج وظائف تدريس اللغة والنحوي والمدخل الذي بني آراءه

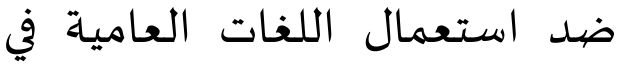
الوقت الحقيقي. و عند سوناردى دويواندونو هو بعض من آراء الخبراء يمكن أن نخرج في الختام وأنّ المدخل الاتصالية هو المدخل الذي مهدف إلى جعل الكفاءة الاتصالية كتعلم اللغة الهدف ووضع إجراءات المهارات اللغوية

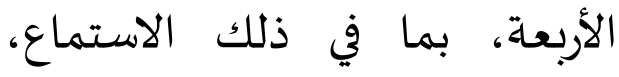
والكلام، والقراءة والكتابة، وتعترف بهاء بالترابط بين اللغة والاتصال، واللغة السؤال في هذا السياق بالطبع اللغة بلة

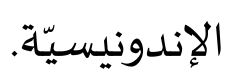

وأنّ المدخل الإنديكه استراتيجية التعليم والتعلم على المان أساس الطلاب النشطة كيف تتعلم، والذي يعرف الآن كالطالب

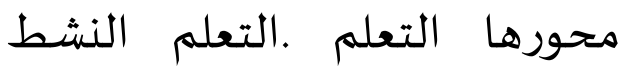
هو تطوير من النظرية "التي (SCL) عبره ديوي وهو التعلم عن طريق العمل. وأنّ ديوي يختلف بشـدة مع دون التعلم بالحفظ عن ظهر القلب. لقد طبّق ديوي مبادئ التعلم بالممارسـة،
في القواعد، و وحب أن يتم إستييلاء قواعد اللغة العبية .(Hijiriyah, 2010)

إنّ تعليم اللغة العربية في جامعة باتوسنكر متجها إلى تعليم الذي مرّ لعرّيم بتعليم الاتصالي والتكاملي واستخدمت باليم بعض طريقة الحديثة في تعليم المهارات

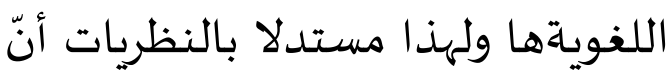
اتجاه التعليم اللغة العربية لابد بنظر بعض المدخلات. ونظرا أنّ في تعليم اللغة العربية هنالك مدخلان اللذان يؤسسان على اتجاه تعليم اللغة خاصا في تعليم اللغة العربية في الجامعة و البيان كما يلي:

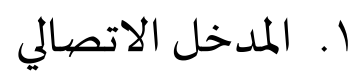
المدخل الاتصالي هو المدلمالي الذي يستند بالفكر على أن القدرة

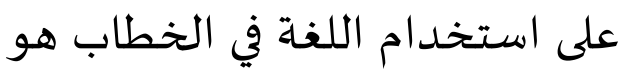
الهدف الذي يجب تحقيقاه في لغة التعلم ووفقا على "هيميس ديل"، تهيل يقول أنّ المدخل الإتصإلى هو إتقان الغريزي التي تنتهي إلى اللغة الأصلية باستخدام وفهم اللغة المعقول الاتصالى أو التفاعل في هذه العملية، وفيما يتعلق بالسياق الاجتماعي. 
على أنّ هذا الاتجاه هو الذي يوحد بعض الجوانب في عملية واحدة. التكاملي ينقسم إلى داخل المواد الدراسية وبين المواد الدراسية. داخل المواد الدراسية بمعنى بعض وبلين الجوانب في حقل واحد متكامل

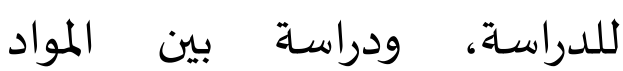
الدراسية هي إدماج المواد من عدة ودراسة بانداد مجالات الدراسـة. - مئه

ورأى الباحث الواقع في قسم تعليم اللغة العربية في هذه الجامعة أنّ التركيز في تعليم اللغة العربية في هذه الجامعة أن يكون تعليم اللغة العربية متكامل ومتصل في تعليم المهارات اللغوية والبيئة اللغوية والأنشطة اللغوية كما ذكر النظرية عن الاتجاه التكاملي. إنّ اتجاه التعليم اللغة العربية على المستوى الجامعي في سومطره قد يكون منظما ومرتبا كما هو الموني المطلوب في الأهداف لتعليم اللغة العربية، وهي أن يكونوا الطلبة قادرين ومستولين على جميع علوم

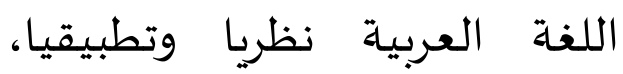
وكذلك لا بد لهم أن يستولوا على

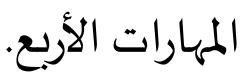

على أن الطلبة بحاجة إلى أن تشـارك

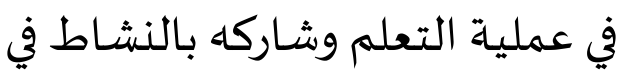
Al-Mohsen عملية التعليم والتعلم (Abeer, 2016)

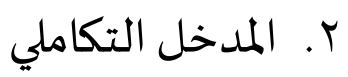
بعد أن لاحظ الباحث وعقد المالم البحث في هذه الجامعة، وجد البد الباحث الحاصل أنّ اتجاه التعليم في جامعة باتوسنكر الإسلامية الحكومية ملائم بنظريات التي ذكر بالئر الباحث من قبل على أنّ منهج تعليم اللغة العربية في هذه الجامعة هو المنهج (KKNI) ويتجـا إلى الإتصالي في بعض المواد الدراسية التي تحتاج إلى إلى المائ استخدام هذا المدخل مثل المادة

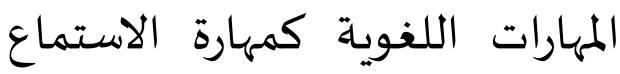
والكلام والقراءة والكتابة. لقد لاحظ الباحث وعقد المقابلة مع بعض المحاضرين وأيضيا أخذ الوثائق في قسم تعليم اللغة العربية، رأى الباحث أنّ قسم تعليم اللغة العربية في هذه الجامعة لباحة سيسعى بكل جهدهم في ترقية تعليم اللغة العربية باستخدم أيضا اتجاه التكاملي في تعليم المواد اللغة

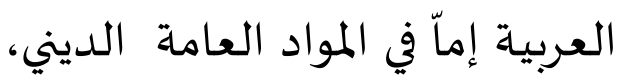


أنّ اتجاه تعليم اللغة العربية في

هذه الجامعة يتجه إلى اتجاه التكاملي

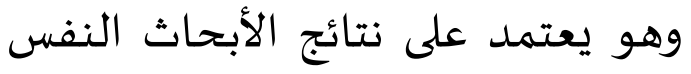

الخلاصية

اللغوية والنفس الإجتماعية وهو يؤكد

إنّ أشكال الاتجاه التعليم

الصلة الوثيقة بين الفنون اللغوية بجامعة باتوسنكر الإسلامية الحكومية

المختلفة حيث إن تعليم إحدى المهارات يتجه إلى الاتجاه التكاملي في المواد يؤثر بالضرورة في المهارات الأخرى، الدراسية مثل المادة المهارات اللغوية. وتنمية كل مهارة لغوية تنمية للمهارات وأنّ قسم تعليم اللغة العربية في هذه الأخرى، كما أنّ أي فنّ منها لا يمكن فهم لفهيه الجامعة يسعى بكل جهدهم في ترقية

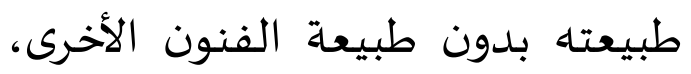
وهذا الاتجاه له تطبيقات عديدة في فيدان الهنون الحري،

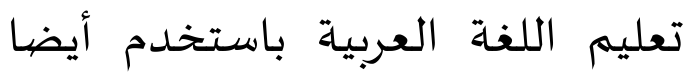

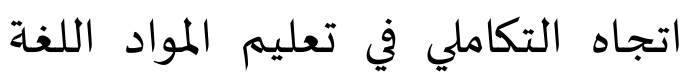
تعليم اللغة وتطوير الاستراتيجيات العربية، وأنّ الرأي الأسـاسي في تعليم وفنيات وإجراءات وطرائق تدريسهامما اللغة العربية في هذه الجامعة أن يكون

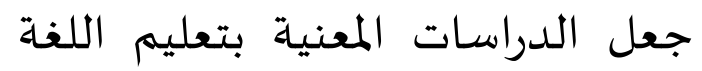

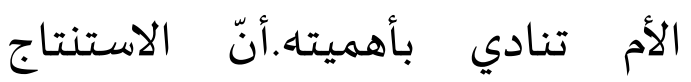
النظري لهذا البحث أنّ اتجاه تعليم اللغة العربية يتجها إلى الاتجاه التكاملي التهاه

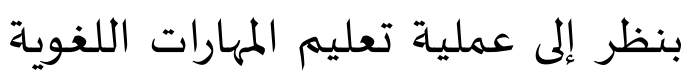

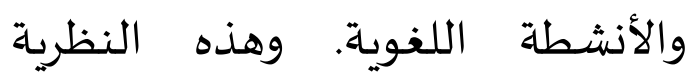
مناسبة لنظرية الاتجاه التكاملي في تعليم اللغة العربية.

المراجع

غباري، ت. أ و شندي، ي. ع. ق، أ. 9. . r. البحث النوعي في التربية تعليم اللغة العربية متكامل ومتصل. و يتجه أيضا هذا قسم تعليم اللغة العربية إلى الاتجاه التكاملي الذي يركز على القواعد و الترجمة وهو تعليم اللغة التحاه

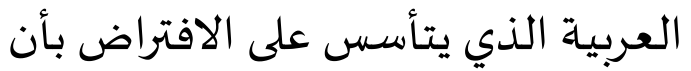
اللغة هي معايير أو قواعد اللغة مأخوذة العربة من نصيوص الفصيح. وهذا الواقع شرح أنّ منهج تعليم في هاتان جامعتان متساويان بعضها ببعض ولكن متفرقة في التركيز بنظر إلى تفرق الرؤية والرسالة وعملية تعليم المهارات اللغوية لفردية اللغوية في تعليم اللغة العربياة. 
القاسمي، م. ع. 9V9 9 ـ اتحجاهات حسليثة

في تعليه اللغتة العربية للناطقة

بلغة أخرى. الرياض: جامعة

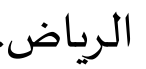

Abeer, A. 2016. Arabic Teachers'

Perception of and Integrated Approach for Teaching Arabic as a Foreign Language in Colleges and Universities in the United States. Dissertation. University of San Fransisco.

Hijiriyah, U. 2016. Paradigma Pembelajaran Bahasa. Bandar Lampung: Pusat Penelitian dan Pengabdian Masyarakat IAIN Raden Intan Lampung.

Miles, M. B. \& Huberman, A. M. 1992. Analisis Data Kualitatif. Jakarta: UIPress.

Mujib, F. 2010. Rekonstruksi Pendidikan Bahasa Arab Dari Pendekatan Konvensional Ke Integratif Humanis. Yogyakarta: Pedagogia.

Retnanto, A. 2009. Bimbingan dan Konseling, Kudus: STAIN Kudus.

Sutanto. 2009. Pengaruh Orientasi Pembelajaran, Kemampuan Produksi, dan Orientasi Pasar terhadap Strategi Bisnis, Ekuitas 13 (4).

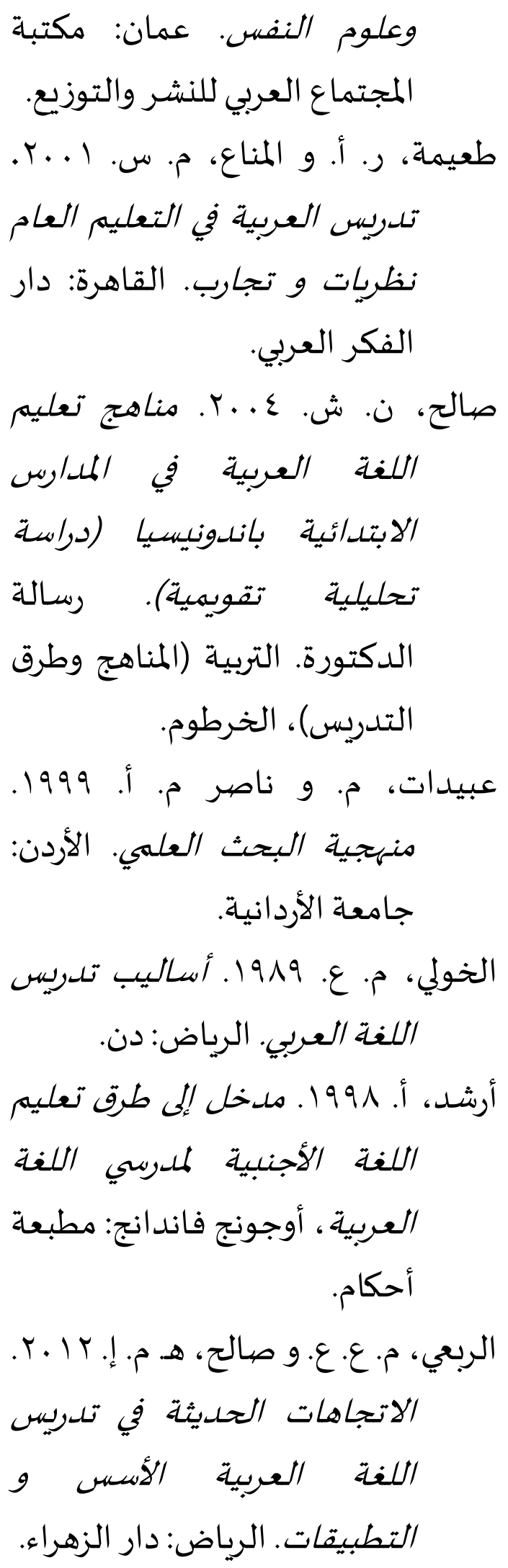

